\title{
Novedades en el uso del barniz de flúor. Reporte de caso
}

\section{Resumen}

Los barnices fluorados se utiliza en la odontologia desde hace mucho tiempo demostrando ser eficaz en la inhibición de la desmineralización del esmalte y promoción de la remineralización del mismo, una presentación tradicional es de color amarillo/naranja que siempre ha sido de cuestionar por los padres de familia si se está
Guido Perona Miguel de Priego ${ }^{1}$

Denisse Aguilar Gálvez ${ }^{1}$

Cecilia Torres Salinas ${ }^{2}$

Relato de caso

\section{A evolução do uso de vernizes de flúor. Relato de caso}

\section{Resumo}

Os vernizes com flúor têm sido utilizados em odontologia há muito tempo, demonstrando eficácia na inibição da desmineralização do esmalte e na promoção de sua remineralização. Sua apresentação tradicional é de cor amarelo/laranja, o que tem sido, sempre, questiona- realizando una profilaxis dental porque aplicar uma sustancia de color sobre los dientes. Se presenta la técnica de um nuevo barniz transparente en un niño de 5 años de edad que presentaba lesiones de manchas blancas y su control a los 21 días.

Palabras clave: Flúor, Carie Dental, Remineralización Dental.

Case report

\section{Developments in the use of varnish flúor. Reporte case}

\begin{abstract}
Fluoride varnishes used in dentistry longtime proving effective in inhibiting enamel demi-

da pelos pais. Apresenta-se a técnica de uso de um novo verniz com flúor transparente em um menino de 5 anos de idade que apresentava lesões de manchas brancas e seu controle aos 21 dias.

Palavras Chave: Flúor, Cárie Dentária, Remineralização Dentária. neralization and promote remineralization of the same, a traditional presentation is yellow / orange color that has always been challenging for parents family if you are performing a den- 
tal prophylaxis because uma colored substance applied on the teeth. Um technique new clear is presented in a child 5 years of age who had white spot lesions and control at 21 days.

Keywords: Fluoride, Dental Caries, Remineralization.

La caries dental en los niños en edad preescolar es un problema significativo de salud pública y en gran medida prevenible, una de las estrategias para combatir esta enfermedad es el uso del flúor en diferentes medios como el agua de consumo humano, pastas dentales, geles dentales, espumas, barnices, enjuagatorios.

El barniz de flúor es una presentación de aplicación profesional para la administración tópica de fluoruro para ayudar a prevenir o controlar dental caries. ${ }^{1}$ El barniz de flúor contiene altas concentraciones de fluoruro, y se encontró una revisión por Marinho et al. ${ }^{2}$ que si se aplica de dos a cuatro veces al año reduce considerablemente la caries dental en los niños, cuando se usa además de cepillarse los dientes regularmente con una pasta dental fluorada.

Durante las últimas décadas, numerosas investigaciones clínicas y de laboratorio han demostrado la eficacia de los barnices fluorados como un agente tópico preventivos de caries dental. Se ha demostrado que la fracción prevenida promedio es del 30\%, ligeramente superior a otros agentes fluorados, además es muy efectivo en grupos de alto riesgo. ${ }^{3}$

Los barnices fluorados también han demostrado inhibición de la desmineralización del esmalte y promoción de la remineralización del mismo. Investigaciones en infantes con alto riesgo de caries, han demostrado que la aplicación de barniz de flúor cada 6 meses es muy útil en la promoción de la remineralización de los dientes afectados. Los barnices fluorados han demostrado también una reducción en la desmineralizacion del esmalte debajo de bandas ortodónticas y brackets; asimismo el barniz de flúor es considerado superior a otras presentaciones y técnicas de aplicación por las siguientes razones ${ }^{3}$ :

- Es más efectivo - mayor reductor de los niveles de caries dental.

- Mayor liberación de flúor y por períodos de tiempo más largos.

- El tiempo de la técnica de aplicación es corta y sencilla.

- El sabor es neutro y bien aceptado.

- No sólo previene la desmineralización, sino que remineraliza las lesiones iniciales de caries (manchas blancas).

La revisión Cochrane de 2009 sobre el barniz de fluoruro de aplicación tópica demostró una eficacia sustancial en la prevención de la caries dental tanto en la dentición decidua y permanente ${ }^{2}$.

Esta evidencia es un componente clave del Departamento de Salud del Reino Unido basada en la evidencia como un conjunto de herramientas para prevención de caries y también para el Programa escocés de Efectividad Clínica Dental (SDCEP) sobre la orientación de prevención y el tratamiento de la caries dental en hijos. En Escocia, el programa nacional de mejoramiento de salud oral "Childsmile", se ha desarrollado para poner en práctica la aplicación del barniz de flúor en la atención dental primaria y guarderías. ${ }^{5}$

En un estudio realizado en la Universidad de Washington ${ }^{6}$, se midió la liberación de flúor invitro después de la aplicación de dos marcas 
comerciales de barniz de flúor. Se usaron muestras de esmalte de dientes primarios sumergidos en una solución de Calcio y Fosfato a un pH de 6.0, previamente cubiertos de una cantidad predeterminada de barniz de flúor. La liberación de flúor se midió cada día/semana hasta que estas muestras dejaron de hacerlo. Como se intuía, una gran cantidad de flúor fue liberada en las primeras 24 horas, y luego esta liberación disminuyó drásticamente. Lo interesante es, que a pesar de la rápida disminución en la liberación de flúor, ésta continuó hasta aproximadamente la semana 16. A pesar de las limitaciones de un estudio in-vitro, se puede concluir que la liberación de flúor continuaría por algunos meses, manteniendo niveles significativos de flúor en el fluido de placa, necesarios para la prevención de caries dental.

Arruda et al. $^{7}$ realizaron un estudio para demostrar la eficacia del barniz fluorado al 5\% en la prevención del incrementos de superficies cariadas en niños con un alto riesgo de caries, las principales conclusiones fueron que encontraron que los incrementos de lesiones de caries fueron más bajos en los niños del grupo con intervención barniz de fluoruro en comparación con el control, con una fracción preventiva del $40 \%$.

A pesar de estos alentadores resultados, se reconoce ampliamente que existe una necesidad de pruebas adicionales en este campo como se indica en la revisión Cochrane, actualmente la tendencia de presentación del barniz de flúor que se comercializa es la presentación transparente/blanco sin variar el contenido de fluoruro de sodio al 5\%, y la posibilidad de dar un valor agregado con otros complementos para mejorar su efectividad, como el Fosfato tricálcico (TCP) Clinpro $^{\mathrm{TM}}$ White Varnish, que es un material inteligente para su uso en productos como el bar- niz de flúor, proporciona biodisponibilidade de iones de calcio y fosfato a los dientes, generando remineralización incluso cuando se utiliza en pequeñas cantidades ${ }^{7}$, diferentes materiales orgánicos se pueden utilizar para adaptar el sistema TCP a una variedad de presentaciones, tales como pasta de dientes, enjuagues bucales y barnices.

Flanigan et $\mathrm{al}^{8}$, realizaron un estudio para determinar la cantidad de calcio, flúor y fósforo que son liberados de un barniz blanco $5 \%$ que contenía TCP (f-TCP) (Clinpro ${ }^{\mathrm{TM}}$ White Varnish) en comparación con un tradicional barniz de fluoruro de sodio $(\mathrm{NaF}) 5 \%$, las concentraciones de calcio, fluoruro y fósforo se midieron en 1, 4, 8 y 24 horas. Los resultados encontrados fue que el barniz blanco con TCP liberó calcio, fluoruro y fosfatos, mientras que el barniz de fluoruro sodio 5\% sólo liberó flúor, concluyendo que el barniz de nueva generación con f-TCP no interfiere en la cantidad de fluoruro liberado y activa la liberación de calcio y el fósforo, convirtiéndolo en un material inteligente.

Asimismo Flanigan et $\mathrm{al}^{9}$, realizaron otro estudio para comparar la remineralización y la resistencia a los efectos de los ácidos proporcionados por un barniz blanco de fluoruro de sodio al 5\% (NaF) con f-TCP (Clinpro ${ }^{\mathrm{TM}}$ White Varnish), otro convencional con fluoruro libre $5 \%(\mathrm{NaF}), \mathrm{y}$ ningún tratamiento, encontraron que las lesiones tratadas con el barniz de flúor blanco $5 \%$ con TCP presentaron una mayor superficie de ganancia de microdureza y una mejor resistencia al ácido que las muestras con tratamiento sólo con barniz de fluoruro de sodio al 5\% (NaF).

Clinpro $^{\mathrm{TM}}$ White Varnish es un líquido blanco, viscoso que contiene $5 \%$ de fluoruro sódico y fosfato tricálcico (f-TCP) cuando se aplica a la superficie del diente en una capa delgada, el 
sistema disolvente (alcohol y agua) se evapora rápidamente, dejando una película, que se adhiere bien a la superficie de los dientes y lentamente libera ion fluoruro, calcio y fosfatos.

La colofonia utilizada en la mayoría de los barnices de flúor es típicamente de color amarillo, aunque el color amarillo color no tiene ningún efecto negativo en la efectividad, muchos pacientes y padres de niños prefieren una película blanca o invisible.

Después de que el Clinpro ${ }^{\mathrm{TM}}$ White Varnish se aplica a los dientes, el producto es prácticamente invisible, otra ventaja es que está envasado en dosis única por unidad para eliminar los problemas de fase separación y la contaminación entre pacientes $^{10}$.

El Clinpro ${ }^{\mathrm{TM}}$ White Varnish Clinpro es tolerante a la humedad y saliva; la saliva activa el componente de TCP protegiendo a la estructura dental conjuntamente con el flúor liberado; $1 \mathrm{ml} \mathrm{de}$ Clinpro $^{\mathrm{TM}}$ White Varnish contiene fluoruro de sodio $50 \mathrm{mg}$, equivalente a 22.6mg (22,600 ppm) de flúor, en una solución a base de alcohol de colofonias modificadas, f-TCP y es endulzado con xilitol que es considerado uno de los elementos preventivos más efectivos atribuyéndosele propiedades importantes en la disminución del biofilm, principalmente porque evita la acumula- ción de colonias de Estreptococos mutans (EsM) y Lactobacilos acidófilos (LaA); es también un estimulante de la secreción salival, factor importante en el mantenimiento de la alcalinidad del $\mathrm{pH}$, además de favorecer el barrido mecánico de las superficies de los dientes, entre otros beneficios ${ }^{11}$.

\section{Dosificación}

$\underline{0,25 \mathrm{ml}}$

- Se utiliza para tratar a pacientes con dentición decidua.

- Se utiliza para tratar a cualquier paciente que requiere la superficie limitada del diente.

\section{$\underline{0.40 \mathrm{ml}}$}

- Se utiliza para tratar a pacientes con dentición mixta que requieren una amplia cobertura área.

- NO utilizar esta cantidad para tratar a pacientes con dentición decidua.

\section{$\underline{0,50 \mathrm{ml}}$}

- Se usa para tratar sólo los pacientes con dentición permanente que requieren una amplia cobertura.
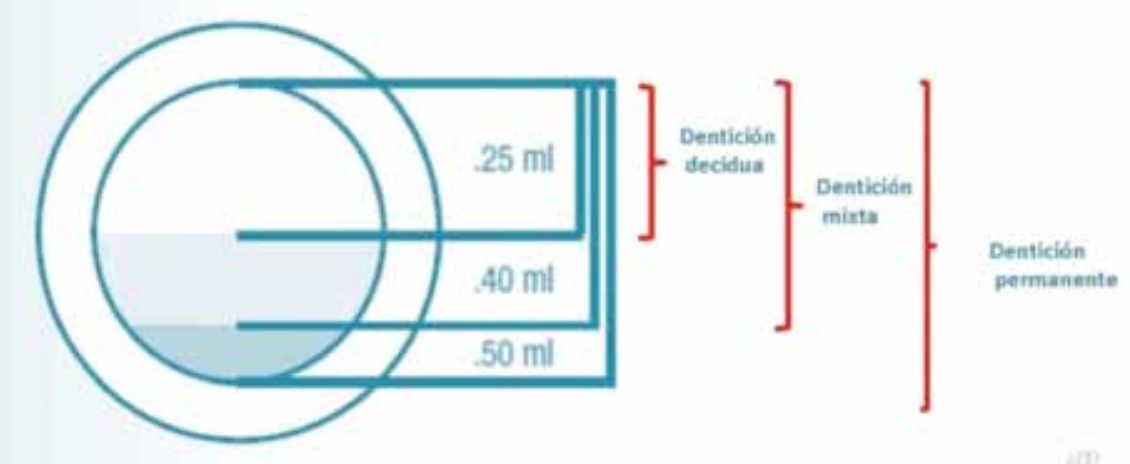

Guía de dosificación de Clinpro ${ }^{\mathrm{TM}}$ White Varnish. 


\section{Reporte de caso clínico}

Se presenta la técnica de la aplicación de un nuevo barniz transparente en un niño de 5 años de edad con alto riesgo de caries. Como antecedentes el paciente sufre de asma bronquial (ahora controlada), usa corticoides cuando se presenta el cuadro de asma, se cepilla los dientes una vez al día; está a cargo de un familiar de la tercera edad (abuela). Se mostró poco colaborador al examen clínico y se observó múltiples lesiones de manchas blancas, biofilm acumulado en las superfícies dentales y lesiones cavitadas.

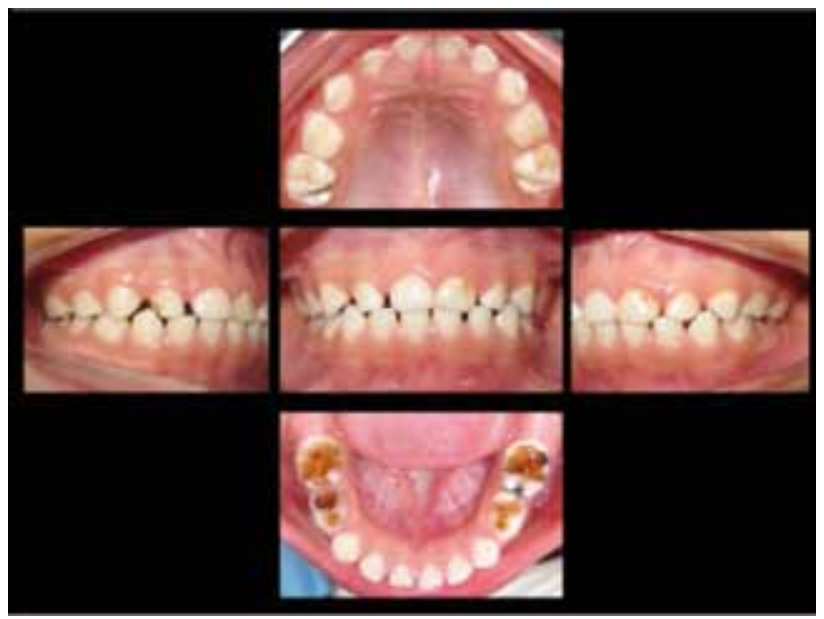

Fig. 1. Selección de paciente: Paciente de alto riesgo múltiples lesiones de caries en superficies libres, oclusal y proximal, presencia de placa blanda, manchas blancas.

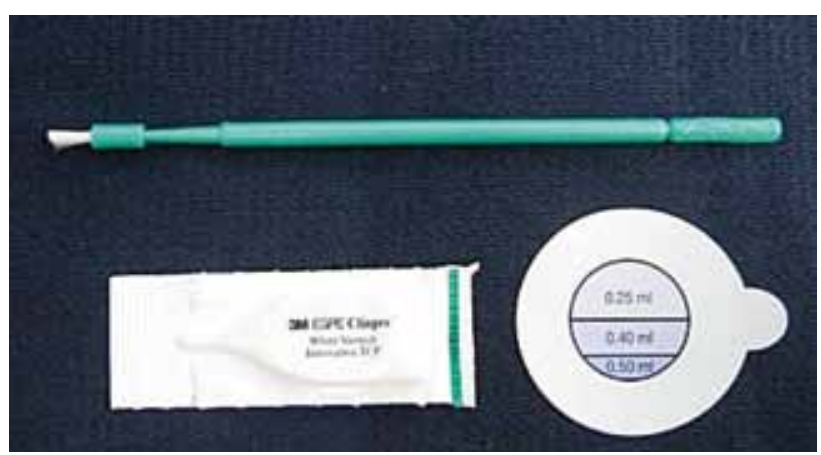

Fig. 1a. Clinpro ${ }^{\mathrm{TM}}$ White Varnish, guía dosificadora, microbrocha.
Se motivó e instruyó al paciente y al familiar cuidador sobre el uso del cepillo dental y el cuidado de la salud bucal.

Se limpió las superfícies dentarias con una gasa húmeda y se aplicó el barniz de flúor en las superfícies dentales, esta aplicación se repitió una vez por semana y se frealizó un control de higiene y de lesiones a los 21 días encontrando cambios visuales de remineralización en las lesiones de manchas blancas donde se aplicó el barniz de flúor.

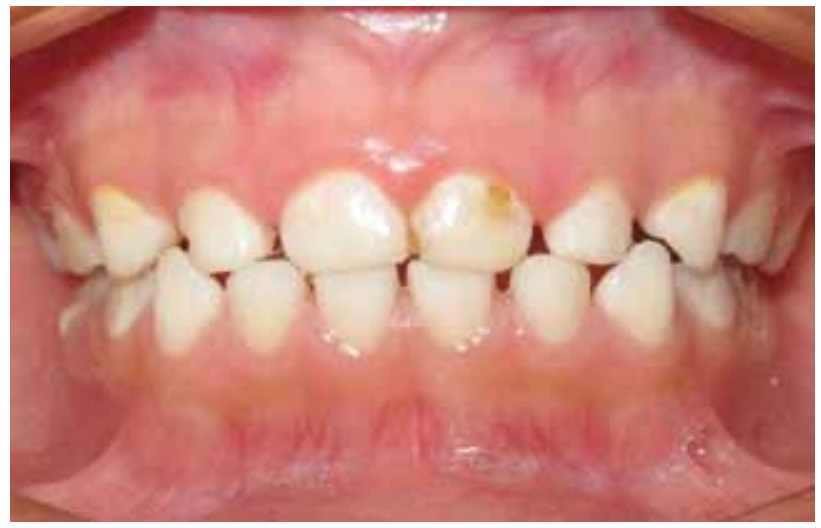

Fig. 2. Examen clínico de paciente.

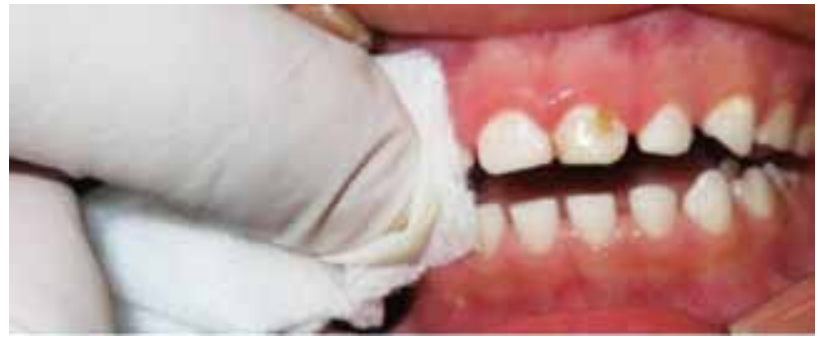

Fig. 3. Limpieza dental con gasa húmeda.

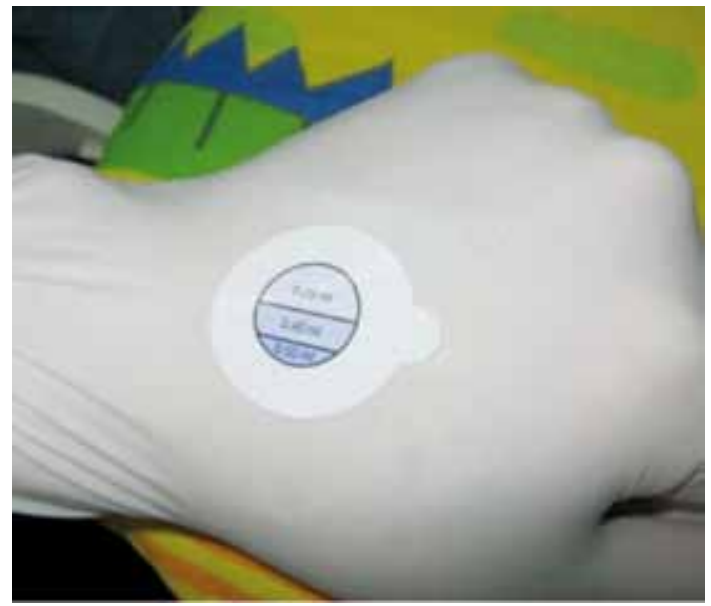

Fig. 4. Sticker con guía dosificadora. 


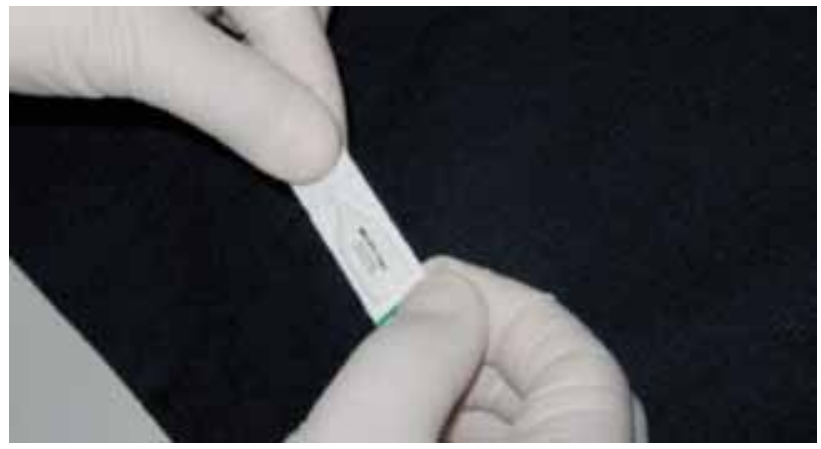

Fig. 5. Dispensador unitario del barniz.

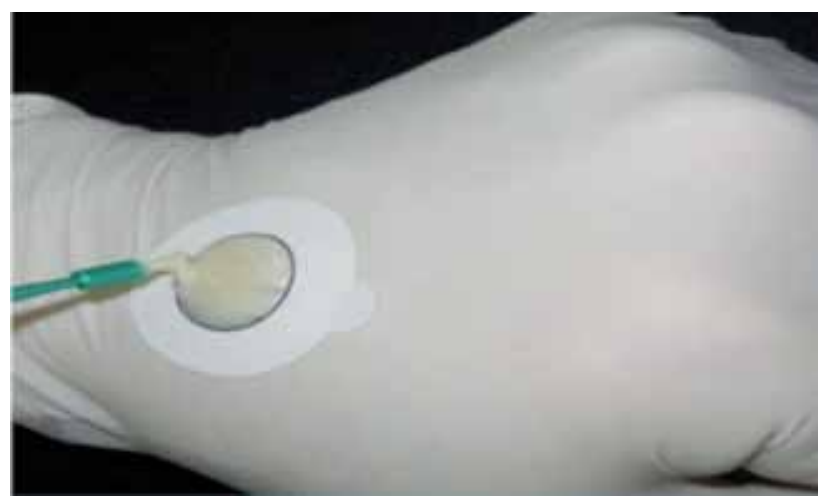

Fig. 7. Mezcla de barniz.

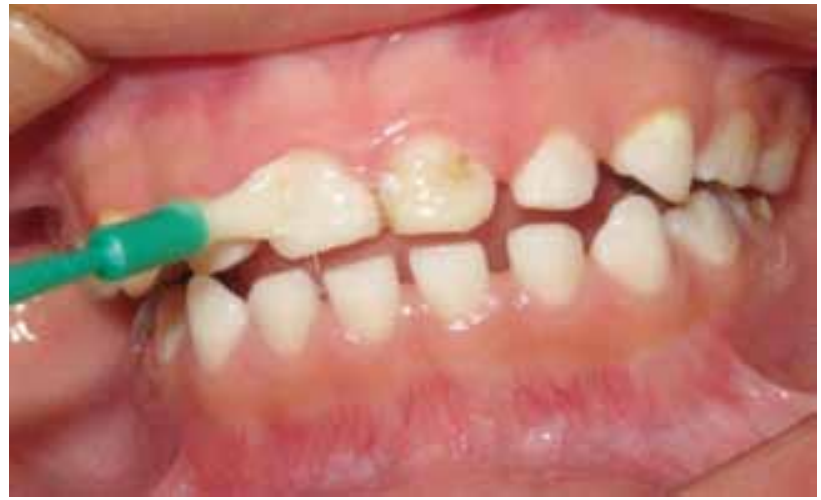

Fig. 9. Aplicación en dientes.

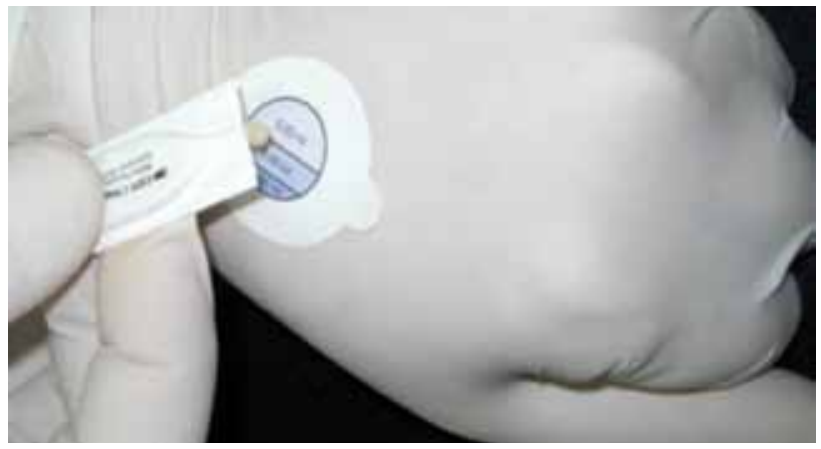

Fig. 6. Colocación de barniz en guía dosificadora.

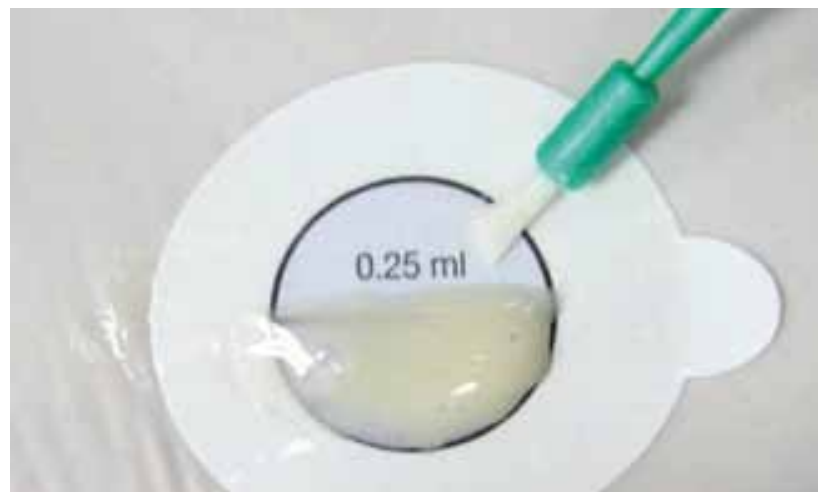

Fig. 8. Dosificación indicada para niños.

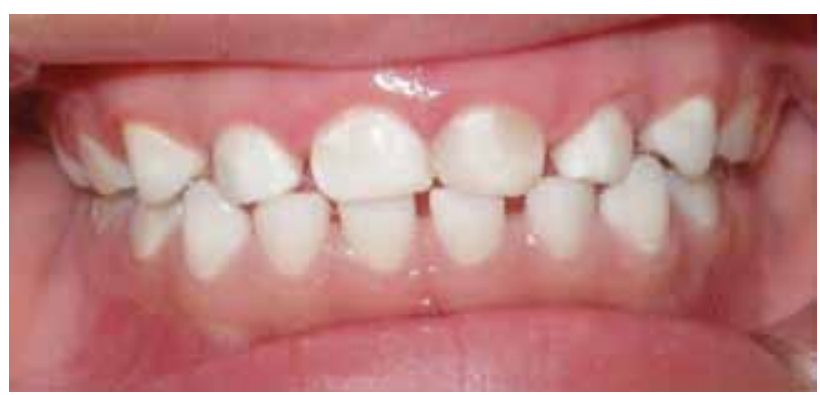

Fig. 10. Control a los 15 días.

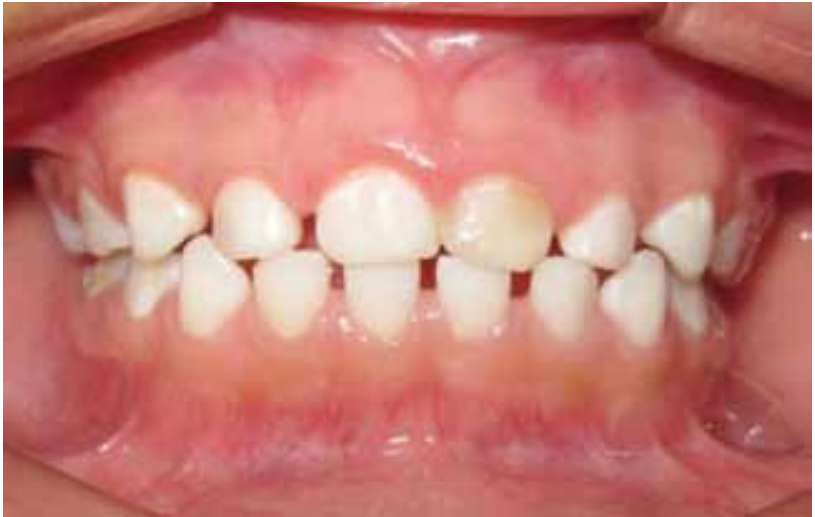

Fig. 11. Control 21 dias. 


\section{Conclusiones}

Todo protocolo de aplicación de barnices de flúor debe estar basado en el riesgo de caries de cada paciente.

El mejor indicador de riesgo de caries es la identificación de manchas blancas y / o antecedentes de caries.
Es clara la evidencia de la eficacia del barniz de flúor en infantes, niños y adolescentes.

También es bueno recordar que deben complementarse las estrategias preventivas como la utilización de sellantes, dieta, cepillado dental con pasta dental fluorada y control periódico.

\section{Referencias}

1. Weintraub JA, Ramos-Gómez F, Jue B,et al. Fluoride varnish efficacy in preventing early childhood caries. J Dent Res 2006; 85: 172-176

2. Marinho VCC, Higgins JPT, Logan S, Sheiham A. Fluoride varnishes for preventing dental caries in children and adolescents. Cochrane Database of Systematic Reviews 2002, Issue 3. Art. No. CD002279.

3. Castillo R. Perona G. Castillo JL Efecto preventivo de los barnices de flúor en la atención primaria de la salud fdimagazine pag 4-5 México 2012

4. Childsmile [website] http: / / www.child-smile.org.uk/

5. Castillo JL, Milgrom P Fluoride Release From Varnishes in Two InVitro Protocols JADA 2004; 135(12):1696-1699.

6. Arruda AO, Senthamarai Kannan R, Inglehart MR, Rezende CT, Sohn W. Effect of 5\% fluoride varnish application on caries among school children in rural Brazil: a randomized controlled trial. Community Dent Oral Epidemiol 2012; 40: 267-276.

7. Pfarrer AM, Karlinsey R Challenges of Implementing New Remineralization Technologies Adv Dent Res 21:79-82, August, 2009

8. Flanigan P. Fitch J. Aeschliman D. New Varnish Releases Fluoride, Calcium and Phosphorous In Vitro J Dent Res 89 (Spec Iss B), 1215, 2010

9. Flanigan P. Vang F. Pfarrer M Remineralization and Acid Resistance Effects of 5\% NaF Varnishes J Dent Res 89 (Spec Iss B), 383, 2010

10. Shen C, Autio-Gold J. Assessing fluoride concentration uniformity and fluoride release from three varnishes. J Am Dent Assoc Feb 2002, 133(2): 176-82.

11. Milgrom P, Ly KA, Roberts MC, Rothen M, Muller H, Yamaguchi DK. Mutans streptococci dose response to Xylitol chewing gum. J Dent Res 2006;137(2):190-6.

Recibido: 11-10-2013

Aceptado: 06-11-2013

Correspondencia: guidoperona54@gmail.com 